\title{
Geometric Analysis of Mesio-buccal Root Canals of Mandibular Molars Prepared by (M-pro), FlexMaster and RaCe File systems: An In Vitro Study
}

\section{Mahmoud Bahaa Eldin Mohammad Gouda ${ }^{1 *}$, Reem Ahmed Lutfy ${ }^{2}$ and Shaimaa Ismail Gawdat ${ }^{3}$}

${ }^{1}$ Masters Student, Faculty of Dentistry, Cairo University, Egypt

${ }^{2}$ Professor of Endodontics, Faculty of Dentistry, Cairo University, Egypt

${ }^{3}$ Associate Professor of Endodontics, Faculty of Dentistry, Cairo University, Egypt

*Corresponding Author: Mahmoud Bahaa Eldin Mohammad Gouda, Masters

Student, Faculty of Dentistry, Cairo University, Egypt.
Received: February 24, 2021

Published: March 22, 2021

(C) All rights are reserved by Mahmoud Bahaa

Eldin Mohammad Gouda., et al.

\begin{abstract}
Aim: The aim of this study was to evaluate and compare 3 file systems; M-Pro, FlexMaster and RaCe in preparing moderately severe curved mesiobuccal canals of human mandibular molars regarding: transportation of the canal, the centering ability and change of radius of canal curvature, using cone-beam computed tomography (СВCT) scanning.

Materials and Methods: A total of fifty-one mesiobuccal root canals of extracted human mandibular first and second molars with the angle of curvature ranging 20-35 (according to Schneider) were collected and divided into three equal groups. After access cavities preparation and working length determination, distal roots with the respective part of the crowns of all samples were sectioned at the furcation level and discarded. Samples were mounted in acrylic resin molds and then CBCT scanning was done pre and post instrumentation using the same scanning parameters. Root canal preparation was done according to the manufacturers' instructions of each NiTi system. The pre and post-images were superimposed using a fusion module of OnDemand 3D App software to measure the dentin thickness at root canal levels $(2,3,5$ and $8 \mathrm{~mm}$ from the tooth apex). Canal transportation and centering ability were recorded according to formula introduced by Gambill., et al. while the change in the radius of canal curvature was recorded following Estrela., et al. Data was tabulated and statistically analyzed.

Results: There was no statistically significant difference among the three rotary systems in canal transportation, centering ability and direction of root canal transportation. Regarding the change of radius in canal curvature, there was a statistically significant difference between pre and post radii of curvature for each file system, but no significant difference was recorded among the three systems.

Conclusion: Under the limitations of this study, it can be concluded that; preparation of curved root canals using M-pro, FlexMaster and RaCe systems was relatively safe. Thus, the new M-pro system could be safely used in preparing curved root canals as it provides a cost-effective alternative.
\end{abstract}

Keywords: M-Pro; FlexMaster; RaCe; Shaping Ability; Geometric Analysis; Mesiobuccal Canals; Mandibular Molars

\section{Introduction}

The main goal of endodontic treatment is prevention and treatment of apical periodontitis [1,2]. Thus, successful endodontic treatment depends mainly on efficient biomechanical preparation and shaping of root canal system [1,3]. During endodontic treatment, it is important to respect the original shape and anatomy of the root canal system; maintaining the position and size of api- 
cal foramen and not changing the original canal curvature $[1,4]$. However, complex anatomy and curvatures of root canal systems in the addition to the traditional stainless-steel instruments make the root canal treatment more difficult [5-7]. Thus, number of endodontic procedural errors such as ledges, perforations and canal transportation, could occur especially on preparing curved root canals [5-7].

Stainless-steel hand instruments are stiff instruments which fail to respect the root canal anatomy, especially with narrow and more curved canals, thus leading to iatrogenic errors during endodontic treatment [5]. Introduction of nickel-titanium rotary instruments was a revolution in the field of endodontics [8]. Nickel-titanium instruments have shown their ability to improve the endodontic treatment and to reduce mishaps occurring due the complex anatomy of the root canal system and old instruments limitations $[8,9]$.

Files with thermomechanical treatment have shown to provide greater flexibility and superior mechanical properties [8-11]. This will lead to better endodontic treatment, increase success rate, decrease endodontic mishaps and obtain better prognosis [8-11]. Recently, many files with thermomechanical treatment have emerged in the market [8-11]

One of the new files introduced to the market is M-pro (Innovative Material and Devices Inc. (IMD), Shanghai, China). It is introduced at much less price compared to other competitors. It is stated by the manufacturer that it is made of X-wire material with special treatment of the raw material, and it can be pre-bended. The manufacturer claims that, it has great elasticity and it is adaptable to most canal anatomies with being well centered in the canal $[12,13]$.

FlexMaster (VDW, Munich, Germany) [14] and RaCe (FKG Dentaire SA, Switzerland) $[15,16]$ systems are conventional nickel-titanium rotary instruments. Previous studies have shown their ability to maintain the original anatomy of the root canal system and they have shown to be safe and effective during preparation of curved canals [17-19].

Cone Beam Computed Tomography (CBCT) is a diagnostic imaging modality used in many studies to evaluate the shaping ability of NiTi rotary systems. It has the advantages of being accurate and providing reproducible date without destruction of the specimens. It also provides high-quality three-dimensional (3D) images for the purposes of endodontic diagnosis, evaluation root canal morphology, fractures and changes in the root canal after instrumentation.

\section{Materials and Methods}

\section{Experimental teeth}

Fifty-one extracted human permanent mandibular first and second molars with average tooth length $20-22 \mathrm{~mm}$ were collected from outpatients surgery clinic, Faculty of Dentistry, Cairo University, Cairo, Egypt, to be used in this study. The collected teeth were thoroughly rinsed under running water, then immersed in $5.25 \%$ sodium hypochlorite for 15 minutes to remove any surface soft tissues or organic debris. Calculus and hard deposits were removed sing ultrasonic scalers.

All teeth were examined by dental operating microscope under 10X magnification to exclude teeth with root caries, cracks, previous restorations or Immature apices.

Periapical radiographs for each tooth were taken in mesiodistal and buccolingual directions to ensure type III mesiobuccal canals, and exclude those with internal or external resorption, calcification, immature canals or endodontically treated canals.

Apical root canal diameter was standardized to size (10 - 15) K-files to allow uniform apical canal preparations using different systems. The mesial root of each tooth was also radiographically checked to ensure that mesiobuccal canal has a curvature within the range of $\left(20^{\circ}-35^{\circ}\right)$ according to Schneider's method [20]. Periapical radiographs were taken for each tooth using digital radiography, with size10 K-file introduced in the mesiobuccal canal and advanced to the apical foramen. A mesiodistal view was taken for each canal using Digora imaging plate and an x-ray machine with exposure parameters; $70 \mathrm{kVp}, 7 \mathrm{~mA}$ and $0.04 \mathrm{sec}$ exposure time. The digital images were saved and then analyzed using the Digimizer Image analysis software (MedCalc Software bvba, Belgium) to determine the mesiobuccal canal curvature according to Schneider's method [20]. Teeth then were stored in saline solution until the time of use.

\section{Sample preparation}

In each tooth, an access cavity was prepared using a high-speed diamond round bur and an Endo-Z bur in a high-speed handpiece with air-water spray coolant. Flattening of crown was carried out using wheel stone high-speed handpiece to standardize the tooth length at $18 \mathrm{~mm}$. The mesiobuccal canal was then located, and 
patency was checked using size $10 \mathrm{~K}$-file. The size $10 \mathrm{~K}$-file was extruded through the apical foramen and the working length was determined by subtracting $1 \mathrm{~mm}$ from the distance measured between the occlusal reference point to the end of the file. Working length of all samples was standardized at $17 \mathrm{~mm}$. Glide path was established using \#15 K-file. Distal roots with the respective part of the crown of all teeth were sectioned at the furcation level using diamond stone under coolant and were discarded. The fifty-one roots were randomly assigned to three equal groups $(n=17)$ according to the NiTi instrument used for root canal preparation; MPro, FlexMaster and RaCe groups. Samples were mounted in plastic square blocks filled with acrylic resin

\section{Pre-instrumentation imaging}

Before canal preparation, a Cone beam computed tomography (CBCT) image was taken for each mold. Images were acquired using iCAT Next Generation scanner (ISI, USA). A scout view was obtained and adjustments were made to ensure that all samples were correctly aligned in the scanner according to the adjustment light beam before acquisition. The machine is supplied with Amorphous Silicon Flat Panel Sensor with Cesium Iodide (CsI) scintillator, 0.5 $\mathrm{mm}$ focal spot size and 14 Bit gray scale resolution. The following operating protocol for the pre-instrumentation, as well as for the post-instrumentation CBCT scans was used: $120 \mathrm{kVp}, 37.07 \mathrm{mAs}$, voxel size $0.125 \mathrm{~mm}$, scanning time $26.9 \mathrm{sec}$, and field of view $4 \mathrm{~cm}$ Height x $16 \mathrm{~cm}$ Diameter.

\section{Pre-operative CBCT measurements}

\section{Radiographic measurements of dentin thickness}

For dentin thickness, OnDemand 3d App software (Cybermed, South Korea) was used. The apex of a tooth was located, and then, four vertical lines of lengths, 2,3,5 and 8 millimeters, were drawn from the apex upwards inside the canal. Axial levels assigned for recording dentin thickness were decided by the end of each of these four lines. At each axial image dentin thickness at mesial and distal aspects of the canal lumen were measured at both scans simultaneously. The procedures were repeated at the four axial levels for each canal. The results were then collected and tabulated for statistical analysis.

\section{Radiographic Measurements of Dentin Thickness}

For radius of canal curvature, the method developed by Estrela., et al. [21] was employed. Sagittal cuts were taken and the canal lumen was located. At each canal lumen, two lines of $6 \mathrm{~mm}$ length, or shorter if necessary, were drawn representing the segments of the canal curvatures, upon which two perpendicular lines were drawn from their centers and extended until they intersect at a central point, which is named circumcenter. The intersection of these perpendicular lines represents the center of canal curvature. Both lines are equal in length and represent the radii of canal curvature.

\section{Root canal instrumentation}

Root canal instrumentation of the three groups was done according to the manufacturers' recommendations for each group. Instrumentation was performed using E-connect pro endo-motor. For each group the same procedures were done. In each canal, glide path was created using size $15 \mathrm{~K}$-file. Irrigation with $5 \mathrm{ml}$ of $2.6 \%$ sodium hypochlorite solution was used before instrumentation using 30-gauge side-vented needle in a plastic syringe. 20/.04 file, $25 \mathrm{~mm}$ (size 20 at the tip and a progressive taper of 0.04 , and a length of $25 \mathrm{~mm}$ ) was first up to the full working length. Irrigation with $5 \mathrm{ml}$ of $2.6 \%$ sodium hypochlorite solution was performed using 30-gauge side-vented needle in a plastic syringe. 25/.06 file, $25 \mathrm{~mm}$ (size 25 at the tip and a progressive taper of 0.06 , and a length of $25 \mathrm{~mm}$ ) was then used up to full working length. Each file was used for 3 canals and then disposed. In all samples, files were used in long and gentle pecking motion (3-4 back and forth gentle strokes) and never advancing more than $1 \mathrm{~mm}$ per second into the canal. Instrument was removed from the canal and cleaned after three pecks; three in and out movements, and the canal was irrigated. MD-ChelCream was used for instrument lubrication.

\section{Post instrumentation evaluation}

After root canal preparation, post-instrumentation CBCT image was taken with the same parameters as the pre-instrumentation image. After acquisition, images were exported and transferred in DICOM format and downloaded via a compact disk for analysis. OnDemand 3D App software (Cybermed, South Korea) was used for the linear and angular measurements.

The fusion module of OnDemand 3D App software was employed to automatically superimpose pre-instrumentation and post-instrumentation scans, hence ensuring measuring dentin thickness at the exact root canal level required. Superimposition sequence was repeated for each sample individually. After fusion of pre-operative and post-operative scans, at any plane, both scans were reconstructed at the same layer. 
The images were evaluated for post-instrumentation changes. The mesio-buccal roots were sliced at 2, 3, 5 and $8 \mathrm{~mm}$ from the apex. Each of the slices was parallel to the horizontal plane on which the mounted tooth was positioned. Each slice was evaluated for:

1. Canal transportation: It is the deviation of the canal axis after instrumentation. Canal transportation was evaluated at each pre-determined levels $(2,3,5$ and $8 \mathrm{~mm})$ in mesial and distal directions, according to the method developed by Gambill., et al. [22] using the following equations:

Mesio-distal transportation: (M1-M2) - (D1-D2), where:

M1 is the distance from the mesial edge of the root to the me sial edge of the un-instrumented canal (the pre-thickness of mesial canal wall).

M2 is the distance from the mesial edge of the root to the mesial edge of the instrumented canal (the post-thickness of mesial canal wall).

D1 is the distance from the distal edge of the root to the distal edge of the un-instrumented canal (the pre-thickness of distal canal wall).

D2 is the distance from the distal edge of the root to the distal edge of the instrumented canal (the post-thickness of distal canal wall).

The result zero of the formula means no transportation, while the positive results indicate mesial transportation, and negative results indicate distal transportation.

2. Canal centering ratio: Canal Centering is the ability of the instrument to remain centered in the canal. Canal centering was evaluated at each pre-determined levels $(2,3,5$ and 8 $\mathrm{mm}$ ) from apex in mesio-distal direction, using the method developed by Gambill., et al. [22] using the following formula: Mesio-distally: (M1-M2) / (D1-D2) or (D1-D2) / (M1-M2), Where: M1, M2, D1 and D2 are the same as described before. If these numbers are not equal, the lower value is considered as the numerator of the ratio. If the result of formula is equal one, this will indicate that the rotary file remained centered (perfect centering ability), while if the result is less than one, this will indicate less centering ability (i.e. less ability of the instrument to keep centralized inside the canal).

3. Change of radius of canal curvature: Root curvature radius was evaluated using the method developed by Estrela., et al [21]. Two lines of equal lengths (6 $\mathrm{mm}$ and shorter if nec- essary) were drawn to the root canal, one line represented the longer continuity of the apical region and the other line represented the middle and cervical thirds. The mid-point of each semi-straight line will be determined. From mid-points, two perpendicular lines were drawn until they meet at a central point, which is named circumcenter. The distance between the circumcenter and the center of each line is the radius of canal curvature [21].

Percentage of change of radius of curvature was calculated from the equation:

(post-instrumentation radius - pre-instrumentation radius) X 100

pre-instrumentation radius

\section{Statistical analysis}

The mean and standard deviation values were calculated for each group in each test. Data were explored for normality using Kolmogorov-Smirnov and Shapiro-Wilk tests, data showed nonparametric (not-normal) distribution. Kruskal Wallis was used to compare between more than two groups in non-related samples. Mann Whitney test was used to compare between two groups in non-related samples. Friedman test was used to compare between more than two groups in related samples. Wilcoxon test was used to compare between two groups in related samples. The significance level was set at $\mathrm{P} \leq 0.05$. Statistical analysis was performed with IBM ${ }^{\circledR}$ SPSS ${ }^{\circledR}$ Statistics Version 20 for Windows.

\section{Results}

Regarding canal transportation, all preparations showed transportation at the nominated levels (Figure 1 and table 1). There was no statistically significant difference among the three rotary systems in canal transportation and centering ability. M-pro group reported transportation at mesio-distal direction that was statistically significantly different at (2 $\mathrm{mm})$ and each of (3 $\mathrm{mm}),(5 \mathrm{~mm})$ and $(8 \mathrm{~mm})$. Significant difference was also reported between $(3$ $\mathrm{mm})$ and ( $8 \mathrm{~mm})$ levels. In RaCe group there was statistically significant difference in mesio-distal transportation between $(2 \mathrm{~mm})$ and each of (3 mm), (5 mm) and $(8 \mathrm{~mm})$, as well as between (3 $\mathrm{mm})$ and $(8 \mathrm{~mm})$ levels.

Regarding overall mesiodistal canal transportation, there was no statistically significant difference among (M-pro), (RaCe) and (Flexmaster) groups (Table 2). 

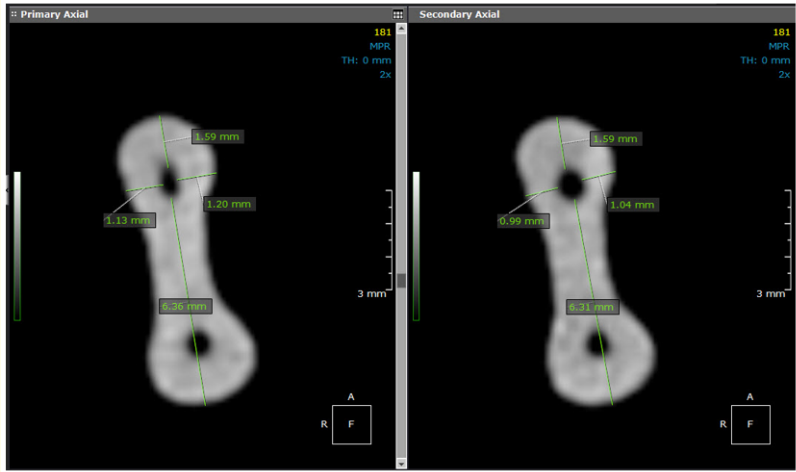

Figure 1: A CBCT image showing measurement of the dentin thicknesses on the axial view of a selected specimen at $3 \mathrm{~mm}$ level.

\begin{tabular}{|l|c|c|c|c|c|c|c|}
\hline \multirow{3}{*}{ Variables } & \multicolumn{7}{|c|}{ MD } \\
& \multicolumn{7}{|c|}{ Canal transportation } \\
\cline { 2 - 7 } & \multicolumn{2}{|c|}{ M-pro } & \multicolumn{2}{|c|}{ RaCe } & \multicolumn{2}{c|}{ FlexMaster } & \multirow{2}{*}{ p-value } \\
\cline { 2 - 7 } & Mean & SD & Mean & SD & Mean & SD & \\
\hline $2 \mathrm{~mm}$ & $0.03^{\mathrm{c}}$ & 0.03 & $0.03^{\mathrm{c}}$ & 0.02 & 0.04 & 0.05 & $0.971 \mathrm{~ns}$ \\
\hline $3 \mathrm{~mm}$ & $0.10^{\mathrm{b}}$ & 0.08 & $0.07^{\mathrm{b}}$ & 0.07 & 0.10 & 0.10 & $0.480 \mathrm{~ns}$ \\
\hline $5 \mathrm{~mm}$ & $0.16^{\mathrm{ab}}$ & 0.15 & $0.10^{\mathrm{ab}}$ & 0.08 & 0.09 & 0.09 & $0.283 \mathrm{~ns}$ \\
\hline $8 \mathrm{~mm}$ & $0.21^{\mathrm{a}}$ & 0.14 & $0.20^{\mathrm{a}}$ & 0.14 & 0.13 & 0.10 & $0.124 \mathrm{~ns}$ \\
\hline p-value & $<0.05^{*}$ & $<0.05^{*}$ & $0.138 \mathrm{~ns}$ & \\
\hline
\end{tabular}

Table 1: The mean, standard deviation (SD) values of mesiodistal canal transportation of different groups.

*: Significant $(\mathrm{p}<0.05)$; ns: Non-Significant $(\mathrm{p}>0.05)$.

\begin{tabular}{|l|c|c|}
\hline \multirow{2}{*}{ Variables } & \multicolumn{2}{|c|}{ Overall Transportation MD } \\
\cline { 2 - 3 } & Mean & SD \\
\hline M-Pro & 0.128 & 0.127 \\
\hline RaCe & 0.100 & 0.106 \\
\hline FlexMaster & 0.092 & 0.091 \\
\hline p-value & \multicolumn{2}{|c|}{$0.258 \mathrm{~ns}$} \\
\hline
\end{tabular}

Table 2: The mean, standard deviation (SD) values of mesiodistal Canal transportation of different groups.

*: Significant $(\mathrm{p}<0.05)$; ns: Non-Significant $(\mathrm{p}>0.05)$.

Regarding the direction of root canal transportation, no significant difference was recorded between the three systems (Table 3).

\begin{tabular}{|c|c|c|c|c|c|c|c|c|}
\hline \multirow[t]{3}{*}{ Variables } & & \multicolumn{7}{|c|}{ MD Canal transportation } \\
\hline & \multicolumn{2}{|c|}{ M-Pro } & \multicolumn{2}{|c|}{ RaCe } & \multicolumn{2}{|c|}{ FlexMaster } & \multirow{2}{*}{$\begin{array}{c}\text { p- } \\
\text { value }\end{array}$} & \\
\hline & $\mathbf{n}$ & $\%$ & $\mathbf{N}$ & $\%$ & $\mathbf{n}$ & $\%$ & & \\
\hline \multirow[t]{3}{*}{$2 \mathrm{~mm}$} & No & 3 & $17.6 \%$ & 2 & $11.8 \%$ & 1 & $5.9 \%$ & \multirow{3}{*}{$0.437 \mathrm{~ns}$} \\
\hline & Mesial & 6 & $35.3 \%$ & 9 & $52.9 \%$ & 4 & $23.5 \%$ & \\
\hline & Distal & 8 & $50 \%$ & 6 & $35.3 \%$ & 12 & $70.6 \%$ & \\
\hline \multirow[t]{3}{*}{$3 \mathrm{~mm}$} & No & 0 & $0 \%$ & 0 & $0 \%$ & 0 & $0 \%$ & \multirow{3}{*}{$0.368 \mathrm{~ns}$} \\
\hline & Mesial & 11 & $64.7 \%$ & 7 & $41.2 \%$ & 8 & $47.1 \%$ & \\
\hline & Distal & 6 & $35.3 \%$ & 10 & $58.8 \%$ & 9 & $52.9 \%$ & \\
\hline \multirow[t]{3}{*}{$5 \mathrm{~mm}$} & No & 0 & $0 \%$ & 0 & $0 \%$ & 4 & $23.5 \%$ & \multirow{3}{*}{$0.109 \mathrm{~ns}$} \\
\hline & Mesial & 6 & $35.3 \%$ & 3 & $17.6 \%$ & 3 & $17.6 \%$ & \\
\hline & Distal & 11 & $64.7 \%$ & 14 & $82.4 \%$ & 10 & $58.8 \%$ & \\
\hline \multirow[t]{3}{*}{$8 \mathrm{~mm}$} & No & 1 & $5.9 \%$ & 0 & $0 \%$ & 1 & $5.9 \%$ & \multirow{3}{*}{$0.587 \mathrm{~ns}$} \\
\hline & Mesial & 10 & $58.8 \%$ & 9 & $52.9 \%$ & 7 & $41.2 \%$ & \\
\hline & Distal & 6 & $35.3 \%$ & 8 & $47.1 \%$ & 9 & $52.9 \%$ & \\
\hline \multicolumn{2}{|l|}{$\mathrm{p}$-value } & \multicolumn{2}{|c|}{$0.253 \mathrm{~ns}$} & \multicolumn{2}{|c|}{$0.278 \mathrm{~ns}$} & \multicolumn{2}{|c|}{$0.052 \mathrm{~ns}$} & \\
\hline
\end{tabular}

Table 3: The frequency of MD canal transportation direction of different groups.

*: Significant $(\mathrm{p}<0.05)$; ns: Non-Significant $(\mathrm{p}>0.05)$.

Concerning the centering ability, there was no statistically significant difference among the three rotary instruments at all levels (Table 4 and 5).

\begin{tabular}{|l|c|c|c|c|c|c|c|}
\hline \multirow{2}{*}{ Variables } & \multicolumn{6}{|c|}{ MD Centering ability } \\
\cline { 2 - 7 } & \multicolumn{2}{|c|}{ M-pro } & \multicolumn{2}{c|}{ RaCe } & FlexMaster & \multirow{2}{*}{ p-value } \\
\cline { 2 - 7 } & Mean & SD & Mean & SD & Mean & SD & \\
\hline $2 \mathrm{~mm}$ & 0.29 & 0.26 & 0.44 & 0.31 & 0.29 & 0.35 & $0.234 \mathrm{~ns}$ \\
\hline $3 \mathrm{~mm}$ & 0.37 & 0.34 & 0.36 & 0.31 & 0.19 & 0.21 & $0.173 \mathrm{~ns}$ \\
\hline $5 \mathrm{~mm}$ & 0.38 & 0.32 & 0.32 & 0.36 & 0.38 & 0.40 & $0.767 \mathrm{~ns}$ \\
\hline $8 \mathrm{~mm}$ & 0.36 & 0.29 & 0.19 & 0.22 & 0.29 & 0.30 & $0.161 \mathrm{~ns}$ \\
\hline p-value & $0.707 \mathrm{~ns}$ & $0.306 \mathrm{~ns}$ & $0.524 \mathrm{~ns}$ & \\
\hline
\end{tabular}

Table 4: The mean, standard deviation (SD) values of mesiodistal Centering ability of different groups.

*: Significant $(\mathrm{p}<0.05)$; ns: Non-Significant $(\mathrm{p}>0.05)$.

Regarding change of radius in canal curvature, there was a statistically significant difference between pre and post radii of curvature for each file system, but no significant difference was recorded among the three systems (Figure 2, table 6 and 7). 
Geometric Analysis of Mesio-buccal Root Canals of Mandibular Molars Prepared by (M-pro), FlexMaster and RaCe File systems: An In Vitro Study

\begin{tabular}{|l|c|c|}
\hline \multirow{2}{*}{\multicolumn{1}{|c|}{ Variables }} & \multicolumn{2}{c|}{ Overall Centering } \\
\cline { 2 - 3 } & Mean & SD \\
\hline M-Pro & 0.35 & 0.30 \\
\hline RaCe & 0.33 & 0.31 \\
\hline FlexMaster & 0.29 & 0.32 \\
\hline p-value & \multicolumn{2}{|c|}{$0.286 \mathrm{~ns}$} \\
\hline
\end{tabular}

Table 5: The mean, standard deviation (SD) values of overall mesiodistal centering in different groups.

*: Significant $(p<0.05)$; ns: Non-Significant $(p>0.05)$.
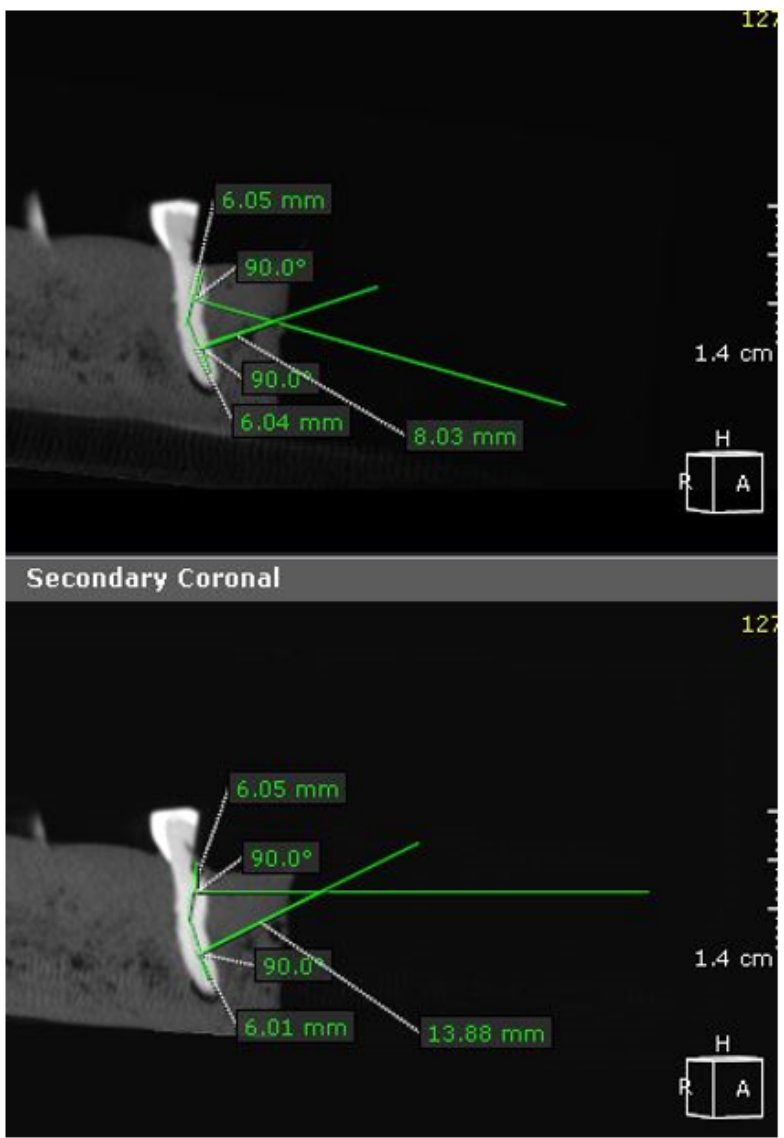

Figure 2: A CBCT image showing measurement of the radii of curvature on the sagittal view of a selected specimen.

\section{Discussions}

The main goal of endodontic treatment is to prevent and treat pulpal or periradicular diseases, and to preserve the natural denti-

\begin{tabular}{|c|c|c|c|c|c|c|}
\hline \multirow{3}{*}{ Variables } & \multicolumn{6}{|c|}{ Change of radius in canal curvature } \\
\hline & \multicolumn{2}{|c|}{ M-pro } & \multicolumn{2}{|c|}{ RaCe } & \multicolumn{2}{|c|}{ FlexMaster } \\
\hline & Mean & SD & Mean & SD & Mean & SD \\
\hline Pre & 20.48 & 7.81 & 17.44 & 6.18 & 15.60 & 4.58 \\
\hline Post & 26.49 & 9.13 & 22.40 & 6.40 & 19.71 & 5.72 \\
\hline p-value & \multicolumn{2}{|c|}{$<0.001^{*}$} & \multicolumn{2}{|c|}{$<0.001^{*}$} & \multicolumn{2}{|c|}{$<0.001^{*}$} \\
\hline
\end{tabular}

Table 6: The mean, standard deviation (SD) values of change of radius in canal curvature in each group.

*: Significant $(\mathrm{p}<0.05)$; ns: Non-Significant $(\mathrm{p}>0.05)$.

\begin{tabular}{|l|c|c|c|}
\hline \multirow{2}{*}{ Variables } & \multicolumn{3}{|c|}{ Total change of radius in canal curvature } \\
\cline { 2 - 4 } & Mean & SD & \% of change \\
\hline M-Pro & 6.01 & 2.74 & $32.16 \%$ \\
\hline RaCe & 4.96 & 1.37 & $31.31 \%$ \\
\hline FlexMaster & 4.11 & 2.54 & $27.17 \%$ \\
\hline p-value & \multicolumn{3}{|c|}{$0.107 \mathrm{~ns}$} \\
\hline
\end{tabular}

Table 7: The mean, standard deviation (SD) values of change of radius in canal curvature in different groups.

*: Significant $(\mathrm{p}<0.05)$; ns: Non-Significant $(\mathrm{p}>0.05)$.

tion when affected by pathosis [1,2]. This is achieved through efficient biomechanical preparation and shaping of root canal system $[1,3]$. Thus, many systems have been introduced to the market to achieve this goal.

The purpose of this study was to analyze geometrical changes in narrow curved mesio-buccal root canals of human extracted mandibular molars when prepared by one of three rotary systems; Mpro, RaCe and FlexMaster rotary systems.

M-pro is a newly introduced NiTi rotary system. It is made of $\mathrm{X}$-wire and it is characterized by a convex triangular cross-section, non-cutting guiding tip and a special heat treatment that allows the files to be pre-bended. The instrument is claimed by the manufacturer to be easy to use as only 2-3 files required in cases, great elasticity and fracture resistance, staying centered in the canal and cost effective [12,13]. M-pro system is introduced at much less price compared to other competitors in the market. FlexMaster system is an austenite NiTi file instrument with a convex triangular cross-section and a rounded, passive safety tip [14]. RaCe is another austenite NiTi system. Each instrument is characterized by a 
triangular cross-section, a non-cutting safety tip, alternating cutting edges and electrochemically polished surface [15]. The three rotary systems share almost similar cross-section, size and taper, that would allow for fair comparison.

In this study, human teeth were used as experimental model in agreement with many authors $[19,23]$. Human teeth were preferred to simulate clinical conditions and to study real instrument performance on dentinal walls. In addition, canals in human teeth vary in their internal anatomy; being irregular with variable degrees of constriction.

Mesio-buccal root canals of extracted human mandibular molars were chosen in the present study in agreement with several authors [24,25]. Mesial root canals are usually curved with variations of curvatures in mesio-distal and bucco-lingual planes. This might not be obvious on periapical radiographs, making them more susceptible to canal transportation, ledge formation and perforation $[26,27]$. Furthermore, those canals have tortuous paths and characteristic concave distal surfaces making them more vulnerable to strip perforation $[28,29]$. Thus, mesio-buccal canals show a high degree of complexity making it challenging to achieve optimal results in terms of shaping ability.

Despite the advantages of using extracted natural teeth, there are many limitations regarding teeth variability. Thus, effort has been done to decrease variability among selected teeth in order to allow high degree of comparability, reproducibility and standardization among the groups $[30,31]$. In the present study, the working length of all samples was standardized to $17 \mathrm{~mm}$ to eliminate the effect of root canal length on the final results [20,27]. Apical root canal diameter was standardized to size (10-15) K files to allow uniform apical canal preparations using different systems. The angle of curvature of mesio-buccal root canals in this study was selected within a range of $20^{\circ}-35^{\circ}$ according to Schneider's method following previous studies $[32,33]$, in order to simulate clinical condition, rather than standardizing definite degree of curvature. Gu., et al. [34] found the average angle of primary curvatures of mesio-buccal canals of mandibular first molars was $24^{\circ}$. In the present study, Schneider's method was used for its simplicity, accuracy and reliability $[35,36]$. Distal roots were resected at the level of bifurcation in order to facilitate inserting the mesial roots in transparent acrylic blocks.

When comparing the shaping ability of different root canal rotary instruments, it is important to reach a standardized post-in- strumentation apical diameter. In the present study, the final apical preparation in all groups was performed with instruments of size 25 and taper 0.06 .

All specimens in the present study were prepared using different NiTi systems according to the manufacturers' instructions, with the aid of EDTA as a lubricant.

In the present study, geometric analysis was assessed using Cone Beam Computer Tomography (CBCT) in terms of three parameters; canal transportation, centering ability and change of radius of curvature. Cone Beam Computed Tomography (CBCT) overcomes the limitations of conventional radiography mainly by producing three-dimensional images. The 3D images allow better visualization of the anatomy. Images produced by CBCT are geometrically accurate and measurements are free from distortion in any plane. In addition, slicing and manipulation of the 3D image could be done by the clinician using CBCT software to allow viewing and visualization of the area of interest in multiple planes, thus eliminating anatomical noise. CBCT has the advantages of being accurate and providing reproducible three-dimensional (3D) images without destruction of the specimens $[37,38]$.

CBCT imaging was used in conjunction with OnDemand 3D App software to allow automatic dentin measurements at the exact preinstrumentation and post-instrumentation root canal levels without human errors. However, this software allows two-dimensional evaluation of the dentin thickness only at selected root canal levels.

In the present study, the parameters used to analyze the geometry of the root canals are canal transportation, centering ability and change of radius of curvature. Canal transportation and centering ability were calculated using the formula given by Gambill., et al. [22], while the radius of curvature was measured using the method given by Estrela., et al. [21]. Transportation is caused when the instrument tends to return to its original straight shape when inserted into a curved root canal. Thus, leading to excessive dentine removal in a certain direction rather than equally in all directions from the main axis of the canal. Centering ratio is the ability of the instrument to remain centered in the prepared canal. The value equal to 1 indicates perfect centering. Dentine thicknesses were measured at 2, 3, 5 and $8 \mathrm{~mm}$ from the apex. The first two levels were established at $2 \mathrm{~mm}$ and $3 \mathrm{~mm}$ from the apex representing the apical third of the canals. At these particular levels, apical canal transportation and zips were found to frequently occur $[39,40]$. The third level was at $5 \mathrm{~mm}$ representing critical level at the middle 
third. The fourth level was at $8 \mathrm{~mm}$ representing beginning of the coronal third. Middle and coronal levels are critical levels prone to stripping especially distally were dangerous zone exists $[41,42]$.

Knowledge of radius of canal curvature allows a more accurate planning of root canal instrumentation and minimizes the impact of the anatomic difficulties and limitations of the endodontic instruments. Change in radius of canal curvature represents straightening of the canal curvature $[43,44]$. Straightening of the canal curvatures is defined as the difference between canal curvature pre- and post-instrumentation [45]. It is a universal finding following instrumentation of curved root canals with various NiTi rotary instruments. The more straightening of canal curvature, the more undesirable weakening of the tooth structure [46]. Radius of canal curvature was measured according to the method developed by Estrela., et al. [21] as it is simple, accurate, reliable and had been employed in different studies $[27,47]$.

Dentin removal during root canal instrumentation is essential because it leads to removal of infected dentin and produces sufficient space for effective irrigation and obturation. It is essential to allow for optimum amount of dentin removal without jeopardizing the endodontic outcome and tooth strength. It was previously reported by Wu., et al. [48] that apical transportation more than 0.3 $\mathrm{mm}$ would compromise the apical seal of root canal filling. In the present study, all transportation values in the three instruments were less than $0.3 \mathrm{~mm}$ in mesio-distal direction. This might indicate the ability of the three rotary instruments to prepare the root canal safely without compromising the apical seal.

There was no statistically significant difference among the three studied rotary instruments at any level at mesio-distal direction. This finding could be explained by the similarity between the three instruments regarding instrumentation technique, rotation motion as well as similarity in size, taper, tip of the three instruments $[5,8]$.

Comparable results for transportation (less than $0.3 \mathrm{~mm}$ ) were previously recorded for RaCe [19,49,50] although in larger sizes but with the same design, proving its efficiency and consistency at all sizes. Similar results for FlexMaster was recorded [51,52] although using larger sizes of the FlexMaster system, but with the same design establishing efficiency and safety at different sizes. On the other hand, inconsistent results were previously reported for RaCe [53] which might be due to difference in the experimental design.
Canal transportation values reported for M-pro group and RaCe were significantly higher at coronal $(8 \mathrm{~mm})$ and at middle $(5 \mathrm{~mm})$ levels in comparison with apical levels ( 2 and $3 \mathrm{~mm}$ ). While FlexMaster group showed slightly higher $3 \mathrm{~mm}$ transportation values compared to $5 \mathrm{~mm}$. In M-pro group, there was statistically significant difference between $(2 \mathrm{~mm})$ and each of ( $3 \mathrm{~mm}),(5 \mathrm{~mm})$ and $(8 \mathrm{~mm})$, as well as between $(3 \mathrm{~mm})$ and $(8 \mathrm{~mm})$ levels. These findings could be explained by the non-cutting guiding tip, special heat treatment, smaller diameter and less metal core of the instrument apically, leading the instrument to be more flexible in the apical area, thus less transportation resulted compared to coronal portion $[5,8]$. In RaCe group, there was statistically significant difference between $(2 \mathrm{~mm})$ and each of ( $3 \mathrm{~mm}),(5 \mathrm{~mm})$ and $(8 \mathrm{~mm})$, as well as between $(3 \mathrm{~mm})$ and $(8 \mathrm{~mm})$ levels. These findings could be explained by the non-cutting, safety tip, alternating cutting edge, and smaller diameter and less metal core of the instrument apically making the instrument more flexible in the apical area, leading to less transportation compared to coronal portion [5,8]. In FlexMaster group, no statistically significant difference was found among different levels.

Comparable results for RaCe group at $3 \mathrm{~mm}$ level were obtained by Saberi and Aramesh [49]. Comparable results for RaCe group were obtained by Al-Sudani and Al-Shahrani [41]. Significant difference was found in the RaCe group between coronal and mid-root level, as coronal transportation value was larger than the mid-root level. In addition, the results for RaCe system were comparable to the results of iRaCe at the study of Hiran-us., et al [54]. iRaCe system have different sequence and ends with a tip diameter size 25 and 0.04 taper but with the same design as the RaCe instruments used in the present study. The readings at levels 2, 3, 5 and 7 were almost similar to the results of the present study. Furthermore, the results for RaCe system were comparable to the results of BioRaCe at the study of Drukteinis., et al [55].

Concerning the results of the overall canal transportation, no statistically significant difference was found among (M-pro), (RaCe) and (FlexMaster) groups. This non-significant difference might be explained by the similarity between the three instruments regarding instrumentation technique, rotation motion as well as similarity in size, taper, tip of the three instruments. Although non-significant difference was found, M-pro was found to show slightly higher overall canal transportation, followed by RaCe and FlexMaster. This might be due to the convex-triangular cross-section [5,8] of the Mpro system compared to RaCe, and the higher rotational speed of M-pro and RaCe systems compared to FlexMaster [56,57]. 
Concerning the results of the direction of canal transportation, the three rotary systems revealed transportation in both direction; mesial and distal, without any statistically significant differences. Results in current study for RaCe group at $5 \mathrm{~mm}$ level at mesiodistal direction were comparable with the results of Drukteinis., et al. [55] at $6 \mathrm{~mm}$ level although using larger size. Comparable results for RaCe group at $5 \mathrm{~mm}$ level were obtained by Saberi and Aramesh [49] at $6 \mathrm{~mm}$ level.

The current results of the centering ability revealed that none of the instruments could remain perfectly centered in the canal. There was no statistically significant difference among the three rotary instruments at all levels. This finding could be explained by the similarity between the three instruments regarding instrumentation technique, rotation motion as well as similarity in the size, taper [5,8]. M-pro system revealed slightly higher overall centering ability compared to RaCe and FlexMaster systems and this might be attributed to the special heat treatment for the M-pro which makes the instrument more flexible and more centered [5,8]. Our results for RaCe group at the coronal and middle levels mesiodistally were consistent with those recorded by Al-Sudani and Al-Shahrani [41]. The study by Schafer and Vlassis [59] demonstrated that the canals instrumented by the RaCe system maintained the original canal curvature significantly better than those prepared with the ProTaper system which is comparable to the results in the present study when comparing to RaCe to FlexMaster. Similar results for FlexMaster group were obtained by Bartols., et al. as it showed less centering ability compared to other systems [60].

Regarding change in radius of canal curvature there was no statistically significant difference found among the three groups. On the other hand, each group significantly resulted in change in the radius of canal curvature after preparation. This significant increase in the radius of canal curvature could be due to the increase of the tip diameter and progressive taper feature of the three rotary systems. The wider diameter of the system at the coronal part compared to the smaller diameter near the tip, leads to decreasing the flexibility which in turn might have resulted in straightening of the canal curvature and increasing the radius of curvature [5,8]. Similar results for the mean of change of radius of canal curvature for FlexMaster system were obtained by Schäfer., et al [61].

From the findings of the present study, it was observed that the three rotary systems were able to effectively shape the severely curved root canals, without significant difference among them in terms of canal transportation, centering ability and radius of curvature. Till present, no NiTi system has proven to be able to prepare canals without any degree of change in canal geometry.

\section{Conclusion}

Under the limitations of this in vitro study, it can be concluded that:

1. Preparation of curved mesio-buccal root canals using M-pro, FlexMaster and RaCe systems was relatively safe.

2. The values of canal transportation of the three rotary systems are considered acceptable.

3. It can be concluded that the new M-pro system is safe in preparing curved root canals as it has shown no significant difference with previously proven safe systems, and it can be a good alternative regarding the cost.

\section{Conflict of Interest}

The authors deny any conflicts of interest in this study.

\section{Bibliography}

1. Schilder H. "Cleaning and shaping the root canal". Dental Clinics of North America 18.2 (1974): 269-296.

2. Trope M. "The vital tooth - its importance in the study and practice of endodontics". Endodontic Top 5.1 (2003): 1.

3. Peters OA., et al. "Contemporary Root Canal Preparation Innovations in Biomechanics" 61 (2017): 37-58.

4. Peters OA and Ficd MS. "Current Challenges and Concepts in the Preparation of Root Canal Systems: A Review (2004).

5. Weine FS and Kelly RF. "The effect of preparation procedures on original canal shape and on apical foramen shape 255-262.

6. Ng Y., et al. "A prospective study of the factors affecting outcomes of nonsurgical root canal treatment: part 1: periapical health (2011): 583-609.

7. Ng Y., et al. "Outcome of primary root canal treatment: systematic review of the literature - Part 2". Influence of Clinical Factors (2008): 6-31.

8. Haapasalo M and Shen Y. "Evolution of nickel-titanium instruments: from past to future". Endodontics Top 29 (2013): 3-17.

9. Bürklein S and Schäfer E. "Critical evaluation of root canal transportation by instrumentation". Endodontics Top 29.1 
(2013): 110-124.

10. Shen Y., et al. "Current challenges and concepts of the thermomechanical treatment of nickel-titanium instruments". Journal of Endodontics 39.2 (2013): 163-172.

11. Gutmann JL and Gao Y. "Alteration in the inherent metallic and surface properties of nickel-titanium root canal instruments to enhance performance, durability and safety: A focused review". International Endodontic Journal 45.2 (2012): 113-128.

12. Innovative Material and Devices Inc. (IMD). (n.d). Rotary File Series. Shanghai, China: Author (2019).

13. http://www.imdmedical.com/\#/Products/About\%20IMD, Accessed on $21 / 4 / 2019$.

14. VDW FlexMaster the rotary NiTi system for every case. Munich, Germany: Author.

15. FKG RaCe family brochure (2017).

16. http://www.fkg.ch/products/endodontics/canal-shapingand-cleaning/race

17. Schäfer E., et al. "Comparison of hand stainless steel and nickel titanium rotary instrumentation: a clinical study". The Journal of Endodontics 30.6 (2004): 432-435.

18. Duran-Sindreu F., et al. "A Comparison of Apical Transportation between FlexMaster and Twisted Files Rotary Instruments". The Journal of Endodontics 38.7 (2012): 993-995.

19. Burkhardt L., et al. "Comparison of the shaping ability of RaCe, FlexMaster, and ProFile nickel-titanium instruments in severely curved root canals". Journal of Clinical and Experimental Dentistry 8.5 (2016): e523-528.

20. Schneider SW. "A comparison of canal preparations in straight and curved root canals (1971).

21. Estrela C., et al. "Method for determination of root curvature radius using cone-beam computed tomography images". Brazilian Dental Journal 19.2 (2008): 114-118.

22. Gambill JM., et al. "Comparison of Nickel-Titanium and Stainless Steel Hand-File Instrumentation Using Computed Tomography 22.7 (1996): 0-6.

23. Race S., et al. "A Comparative Study of Shaping Ability of four Rotary Systems". Acta stomatologica Croatica 49.8 (2015): 285-
293.

24. Mahran AH and AboEl-Fotouh MM. "Comparison of effects of ProTaper, HeroShaper, and Gates Glidden Burs on cervical dentin thickness and root canal volume by using multislice computed tomography". The Journal of Endodontics 34.10 (2008): 1219-1222.

25. Vahid A., et al. "A comparative study of four rotary NiTi instruments in preserving canal curvature, preparation time and change of working length". Australian Endodontic Journal 35.2 (2009): 93-97.

26. Hashem AAR., et al. "Geometric analysis of root canals prepared by four rotary NiTi shaping systems". The Journal of Endodontics 38.7 (2012): 996-1000.

27. Marzouk AM and Ghoneim AG. "Computed tomographic evaluation of canal shape instrumented by different kinematics rotary nickel-titanium systems". The Journal of Endodontics 39.7 (2013): 906-909.

28. Hargreaves Kenneth M CS. “Cohen's pathways of the pulp, 11th edition". British Dental Journal 210 (2016): 223-349.

29. Peters OVEA., et al. "Mechanical preparation of root canals : shaping goals, techniques and means 10 (2005): 30-76.

30. Veltri M., et al. "In vitro comparison of shaping abilities of ProTaper and GT rotary files". The Journal of Endodontics 30.3 (2004): 163-166.

31. Iqbal MK., et al. "Comparison of apical transportation between ProFile and ProTaper NiTi rotary instruments". International Endodontic Journal 37.6 (2004): 359-364.

32. Wu X-C and Zhu Y-Q. "Geometric analysis of root canals prepared by single twisted file in three different operation modes". European Journal of Dentistry 8.4 (2014): 515-520.

33. Mittal A., et al. "Comparative Assessment of Canal Transportation and Centering Ability of Reciproc and One Shape File Systems Using CBCT-An In Vitro Study". JBR Journal of Clinical Diagnosis and Research Open Access 11.4 (2017): ZC31-ZC34.

34. Gu Y., et al. "Root canal morphology of permanent three-rooted mandibular first molars: Part II--measurement of root canal curvatures". The Journal of Endodontics 36.8 (2010): 13411346. 
35. Arıcan Öztürk B., et al. "Cone-Beam Computed Tomographic Analysis of Shaping Ability of XP-endo Shaper and ProTaper Next in Large Root Canals". The Journal of Endodontics (2020).

36. Webber M., et al. "Higher Speed and No Glide Path: A New Protocol to Increase the Efficiency of XP Shaper in Curved CanalsAn In Vitro Study". The Journal of Endodontics 46.1 (2020): 103109.

37. Sharma S., et al. "CBCT diagnosis and endodontic management of a maxillary first molar with unusual anatomy of two palatal canals: A case report". Journal of Conservative Dentistry: JCD 17.4 (2014): 396-399.

38. Patel S., et al. "Cone beam computed tomography in Endodontics - a review". International Endodontic Journal 23 (2014): 3-15.

39. American Association of Endodontists Glossary of Endodontic Terms". 7th edition. Chicago: AAE (2003).

40. Moazzami F., et al. "Cone-Beam Computed Tomography Assessment of Root Canal Transportation by Neoniti and Reciproc Single-File Systems". The Iranian Endodontic Journal 11.2 (2016): 96-100.

41. Al-Sudani D and Al-Shahrani S. "A Comparison of the Canal Centering Ability of ProFile, K3, and RaCe Nickel Titanium Rotary Systems". Journal of Endodontics 32.12 (2006): 1198-1201.

42. Abou-Rass M and Jastrab RJ. "The use of rotary instruments as auxiliary aids to root canal preparation of molars". Journal of Endodontics 8.2 (1982): 78-82.

43. Paque F., et al. "Comparison of root canal preparation using RaCe and ProTaper rotary Ni-Ti instruments". International Endodontic Journal 38.1 (2005): 8-16.

44. Schafer E., et al. "Comparative study on the shaping ability and cleaning efficiency of rotary Mtwo instruments. Part 2. Cleaning effectiveness and shaping ability in severely curved root canals of extracted teeth". International Endodontic Journal 39.3 (2006): 203-212.

45. Burklein S., et al. "Shaping ability and cleaning effectiveness of two single-file systems in severely curved root canals of extracted teeth: Reciproc and WaveOne versus Mtwo and ProTaper". International Endodontic Journal 45.5 (2012): 449-461.
46. Schafer E and Schlingemann R. "Efficiency of rotary nickeltitanium K3 instruments compared with stainless steel hand K-Flexofile. Part 2. Cleaning effectiveness and shaping ability in severely curved root canals of extracted teeth". International Endodontic Journal 36.3 (2003): 208-217.

47. Capar ID., et al. "Comparative study of different novel nickeltitanium rotary systems for root canal preparation in severely curved root canals". The Journal of Endodontics 40.6 (2014): 852-856.

48. Wu MK., et al. "Leakage along apical root fillings in curved root canals. Part I: effects of apical transportation on seal of root fillings". The Journal of Endodontics 26.4 (2000): 210-216.

49. Saberi E and Aramesh B. "Computed tomography evaluation of root canal transportation using ProTaper, Race and Sendoline rotary systems: an ex vivo study". Clinical, Cosmetic and Investigational Dentistry 10 (2018): 93-98.

50. Mamede-Neto I., et al. "Root Canal Transportation and Centering Ability of Nickel-Titanium Rotary Instruments in Mandibular Premolars Assessed Using Cone-Beam Computed Tomography". The Open Dentistry Journal 11.1 (2017): 71-78.

51. Moore J., et al. "A micro-computed tomographic evaluation of apical root canal preparation using three instrumentation techniques". International Endodontic Journal 42.12 (2009): 1057-1064.

52. García M., et al. "A Comparison of Apical Transportation between ProFile and RaCe Rotary Instruments". Journal of Endodontics 38.7 (2012): 990-992.

53. Honardar K., et al. "Cone-beam Computed Tomographic Assessment of Canal Centering Ability and Transportation after Preparation with Twisted File and Bio RaCe Instrumentation". The Journal of Dentistry 11.4 (2014): 440-446.

54. Hiran-us S., et al. "Shaping ability of ProTaper NEXT, ProTaper Universal and iRace files in simulated S-shaped canals". Australian Endodontic Journal 42.1 (2016): 32-36.

55. Drukteinis S., et al. "Shaping ability of BioRace, ProTaper NEXT and Genius nickel-titanium instruments in curved canals of mandibular molars: a MicroCT study". International Endodontic Journal 52.1 (2019): 86-93. 
56. Karagöz-Küçükay I., et al. "Effect of Rotational Speed on Root Canal Preparation with Hero 642 Rotary Ni-Ti Instruments". The Journal of Endodontics 29.7 (2003): 447-449.

57. Yildiz ED., et al. "Effect of instrument speed when used in reciprocating motion on root canal transportation and centering ability". Journal of Conservative Dentistry 20.4 (2017): 234-236.

58. Garcia Filho PF., et al. "Danger zone in mandibular molars before instrumentation: an in vitro study". Journal of Applied Oral Science 11 (2003): 324-326.

59. Schafer E and Vlassis M. "Comparative investigation of two rotary nickel-titanium instruments: ProTaper versus RaCe. Part 2. Cleaning effectiveness and shaping ability in severely curved root canals of extracted teeth". International Endodontic Journal 37.4 (2004): 239-248.

60. Bartols A., et al. "Assessment of Different Root Canal Preparation Techniques with Rotary Nickel-Titanium Instruments by Novice Students". The Journal of Dentistry 6.3 (2018): 46.

61. Schafer E., et al. "Influence of different types of automated devices on the shaping ability of rotary nickel-titanium FlexMaster instruments". International Endodontic Journal 38.9 (2005): 627-636.

\section{Assets from publication with us}

- Prompt Acknowledgement after receiving the article

- Thorough Double blinded peer review

- Rapid Publication

- Issue of Publication Certificate

- High visibility of your Published work

Website: www.actascientific.com/

Submit Article: www.actascientific.com/submission.php

Email us: editor@actascientific.com

Contact us: +919182824667 